\title{
QUANTUM GROUPS AND DIFFERENTIAL FORMS
}

\author{
SWAPNEEL MAHAJAN
}

\begin{abstract}
We construct a quantum semigroup and an algebra of forms appropriate for the generalised homological algebra of $N$-complexes [8]. This is an analogue to the picture for usual homological algebra, where one has the quantum general linear group [9] and the differential forms constructed by Wess and Zumino [17]. The two pictures are different and we explain why the dichotomy arises.
\end{abstract}

\section{Contents}

1. Introduction

2. The categorical setting

3. The free associative picture

4. Imposing relations

5. Imposing more relations

6. Completing the proof of Theorem 1

7. Connection to the Yang Baxter equation

Acknowledgements $\quad 13$

References

\section{INTRODUCTION}

In the classical situation, for a manifold $K$, one defines an algebra of differential forms $\Omega(K)$. It is a graded algebra equipped with a graded derivation $d$ of degree one satisfying $d^{2}=0$. By a graded derivation, we mean that $d$ satisfies the Leibniz rule for the (wedge) product on $\Omega(K)$. If we restrict ourselves to a single coordinate chart or assume that $K=\mathbb{R}^{n}$ then the general linear group $G L_{n}$ acts on $\Omega(K)$. Dually, the ring of regular functions on $G L_{n}$, which we denote by $G L(n)$, coacts on $\Omega(K)$. Recall that $G L(n)$ has the structure of a Hopf algebra that is commutative but not cocommutative.

In the quantum situation, one deforms the above Hopf algebra and constructs a quantum group $G L_{q}(n)$ which coacts on a suitably deformed algebra of differential forms $\Omega\left(K_{q}\right)$. The Hopf algebra $G L_{q}(n)$ is known in the literature as the quantum general linear group $[9,12,13]$. Its connection with differential forms first appeared in the work of Wess and Zumino [17]. For a later survey, see the paper by Manin

Correspondence: School of Mathematics, Tata Institute of Fundamental Research, Dr. Homi Bhabha Road, Mumbai 400 005, India; E-mail: smahajan@math.tifr.res.in.

2000 Mathematics Subject Classification. Primary 12H05, 16W30. Secondary 18G99.

Key words and phrases. generalised homology; FRT construction; Hecke algebra; Yang Baxter equation. 
[14]. For us, a quantum group (resp. quantum semigroup) is a Hopf algebra (resp. bialgebra) which is neither commutative nor cocommutative.

In this paper, we look at an analogue to the above situation. We start with an algebra of differential forms $\Omega(A)$, where the differentials $d x_{i}$ commute instead of skew-commute and then deform it to $\Omega\left(A_{q}\right)$. These considerations are motivated by the generalised homological algebra of $N$-complexes [8]. Throughout this paper, we assume that $N \geq 2$ and $k$ is any field.

1.1. The commutative setting. Let $V$ be the vector space over $k$ with basis $S=\left\{x_{1}, \ldots, x_{n}\right\}$.

Definition 1.1. Let $A=k\left[x_{1}, \ldots, x_{n}\right]$ be the free commutative algebra in $n$ variables $x_{1}, \ldots, x_{n}$. Define the algebra of forms

$$
\Omega(A)=k\left[x_{1}, \ldots, x_{n}, d x_{1}, \ldots, d x_{n}\right] .
$$

There is a differential $d: \Omega(A) \rightarrow \Omega(A)$ such that $d\left(x_{i}\right)=d x_{i}$ and $d\left(d x_{i}\right)=0$ for $1 \leq i \leq n$ and

$$
d(u v)=d(u) v+u d(v) .
$$

In other words, the differentials $d x_{i}$ commute and the differential $d$ is an ordinary derivation. So the differential $d$ on $\Omega(A)$ does not satisfy $d^{2}=0$.

Definition 1.2. Let the (matrix) bialgebra $M$ be the free commutative algebra on the generators $t_{i j}$ for $1 \leq i, j \leq n$ with the coproduct given by $\Delta: M \rightarrow M \otimes M$ with the generator $t_{i j} \mapsto \sum_{k=1}^{n} t_{i k} \otimes t_{k j}$.

The space $\Omega(A)$ has a coaction of the bialgebra $M$, specified on the generators by

$$
x_{i} \mapsto \sum_{k=1}^{n} t_{i k} \otimes x_{k} \text { and } d x_{i} \mapsto \sum_{k=1}^{n} t_{i k} \otimes d x_{k} .
$$

1.2. The quantum setting. The amazing part is that $\Omega(A)$ admits a two parameter deformation which we may denote $\Omega_{s}\left(A_{q}\right)$ along with a quantum (matrix) semigroup $M_{q}$ that coacts on it, see Theorem 1. The bialgebra $M_{q}$ is a two parameter deformation of $M$. These deformations can be constructed systematically. We will give more details in Sections 3-6. The discussion so far can be summarised in the following table.

TABLE 1.

\begin{tabular}{|l|c|c|c|c|}
\cline { 2 - 5 } \multicolumn{1}{c|}{} & \multicolumn{2}{c|}{ Standard picture } & \multicolumn{2}{c|}{ Our picture } \\
\hline Classical & $G L(n)$ & $\Omega\left(\mathbb{R}^{n}\right)$ & $M$ & $\Omega(A)$ \\
\hline Quantum & $G L_{q}(n)$ & $\Omega\left(\mathbb{R}_{q}^{n}\right)$ & $M_{q}$ & $\Omega\left(A_{q}\right)$ \\
\hline
\end{tabular}

In the rest of this section, we give the definitions of $\Omega_{s}\left(A_{q}\right)$ and $M_{q}$ using generators and relations and then discuss the precise relation between the two. The bialgebra $M_{q}$ can be seen as a special case of the FRT construction due to Faddeev, Reshetikhin, and Takhtajan [7]. We treat this and the relation to the Yang Baxter equation in Section 7. 
1.3. The algebra of forms. We now define an algebra of forms $\Omega\left(A_{q}\right)$ that depends on two parameters $q$ and $s$. For simplicity, we suppress the second parameter $s$ from the notation. First let

$$
A_{q}=k\left\{x_{1}, \ldots, x_{n}\right\} /\left(x_{i} x_{j}-q x_{j} x_{i} \text { for } i>j\right)
$$

be a deformation of the symmetric algebra $A=k\left[x_{1}, \ldots, x_{n}\right]$. It is sometimes referred to as the Manin $n$-plane. Next let

$$
d A_{q}=k\left\{d x_{1}, \ldots, d x_{n}\right\} /\left(d x_{i} d x_{j}-q d x_{j} d x_{i} \text { for } i>j\right) .
$$

Note that $d A_{q}$ is not a deformation of the exterior algebra. Instead it is a deformation of an algebra where the differentials commute instead of skew-commute. In other words, $A_{q} \cong d A_{q}$ as algebras.

Definition 1.3. We define the algebra of forms

$$
\Omega\left(A_{q}\right)=A_{q} * d A_{q},
$$

as the free product of $A_{q}$ and $d A_{q}$, subject to the following twist relations.

$\left(1^{\prime}\right) d x_{i} x_{j}=q x_{j} d x_{i}+(s-1) x_{i} d x_{j}$ for $i>j$.

(1") $d x_{j} x_{i}=s q^{-1} x_{i} d x_{j}$ for $i>j$.

(2) $d x_{k} x_{k}=s x_{k} d x_{k}$.

It is graded by the number of differential symbols. Furthermore, there is a differential $d: \Omega\left(A_{q}\right) \rightarrow \Omega\left(A_{q}\right)$ such that $d\left(x_{i}\right)=d x_{i}$ and $d\left(d x_{i}\right)=0$ for $1 \leq i \leq n$ and the $s$-Leibniz rule holds:

$$
d(u v)=d(u) v+s^{|u|} u d(v) .
$$

Here $|u|$ is the degree of $u$ with respect to the grading on $\Omega\left(A_{q}\right)$. Though $d^{2}$ is zero on the generators $x_{i}$, we cannot conclude $d^{2}=0$. This is because the Leibniz rule has been skewed by the parameter $s$ rather than the usual $(-1)$. Note that setting $q=s=1$, gives us the basic object $\Omega(A)$ in Definition 1.1.

Remark. The object $\Omega\left(A_{q}\right)$ is a graded $s$-differential $g$-algebra, with generating set $S=\left\{x_{1}, \ldots, x_{n}\right\}$. This notion is explained in Section 2 .

1.4. The order of the differential $d$ and $N$-complexes. Let the field $k$ have characteristic 0 . Then, for the basic object $\Omega(A)$, the differential $d$ has infinite order, that is, $d^{N}=0$ does not hold for any $N$. And for generic $s$ and $q$, the same is true for the deformed object $\Omega\left(A_{q}\right)$. However if we choose the parameter $s$ to be a primitive $N$ th root of unity then it follows from equation (1.2) that $d^{N}=0$ (see Lemma 1). This is the relevance of the paper to the more general homological algebra of $N$-complexes [8]. For the basic theory, also see [3, 4, 10]. For an overview of the mathematical theory and applications to physics, see the paper of DuboisViolette [5] and the references therein. Ideas more specific to differential forms can be found in $[1,2,6,11]$. Early references to the identity $d^{N}=0$ are $[15,16]$.

This behaviour of the differential is in contrast to the standard picture where $d^{2}=0$ holds for the usual algebra of differential forms $\Omega\left(\mathbb{R}^{n}\right)$ as well as for the deformed one $\Omega\left(\mathbb{R}_{q}^{n}\right)$. 
1.5. The quantum semigroup. The covariance of the algebra of forms $\Omega\left(A_{q}\right)$ leads us to the quantum semigroup $M_{q}$ which we now define. First let $\tilde{M}$ be the free associative algebra generated by the symbols $t_{i j}$ for $1 \leq i, j \leq n$ with the coproduct given by $\Delta: \tilde{M} \rightarrow \tilde{M} \otimes \tilde{M}$ with the generator $t_{i j} \mapsto \sum_{k=1}^{n} t_{i k} \otimes t_{k j}$.

Definition 1.4. Define the quantum semigroup $M_{q}$ to be the bialgebra $\tilde{M}$ subject to the following relations.

(i') $q^{2} t_{j k} t_{i l}=s t_{i l} t_{j k}$ for $i>j, k>l$.

(i") $q t_{i k} t_{j l}=q t_{j l} t_{i k}+(s-1) t_{i l} t_{j k}$ for $i>j, k>l$.

(ii) $t_{i k} t_{j k}=q t_{j k} t_{i k}$ for $i>j$. (column relations)

(iii) $t_{i l} t_{i k}=q s^{-1} t_{i k} t_{i l}$ for $k>l . \quad$ (row relations)

Note that $M_{q}$ is a two parameter deformation of the bialgebra $M$ in Definition 1.2. We obtain the later by setting $q=s=1$.

1.6. The covariance of $\Omega\left(A_{q}\right)$. Now we explain the connection between $\Omega\left(A_{q}\right)$ and $M_{q}$. We think of $\Omega\left(A_{q}\right)$ as a noncommutative space and $M_{q}$ as its space of endomorphisms.

Theorem 1. The algebra of forms $\Omega\left(A_{q}\right)$ is a $M_{q}$ comodule algebra, that is, there is a coaction

$$
\delta: \Omega\left(A_{q}\right) \rightarrow M_{q} \otimes \Omega\left(A_{q}\right),
$$

which is a morphism of algebras. The coaction preserves $V$, the space spanned by $x_{1}, x_{2}, \ldots, x_{n}$. And, the differential $d: \Omega\left(A_{q}\right) \rightarrow \Omega\left(A_{q}\right)$ is a morphism of $M_{q}$ comodules. In other words, the following diagram commutes.

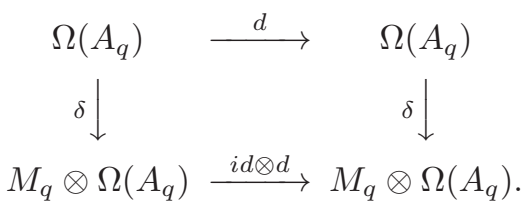

Furthermore, the bialgebra $M_{q}$ is universal with respect to these properties.

The coaction of $M_{q}$ on the generators of $\Omega\left(A_{q}\right)$ is given by equation (1.1). In principle, the theorem can be verified directly from the definitions. But that is not a good approach because it does not tell us how to construct $\Omega\left(A_{q}\right)$ and $M_{q}$ in the first place. We now address this question.

1.7. Method of construction. The theorem will be proved in three stages (Sections 3-6). The method is to construct $\Omega\left(A_{q}\right)$ and $M_{q}$ simultaneously using the properties in the theorem as starting constraints. So when we are done, these properties would automatically hold. But in any case, we need to start somewhere, preferably with a situation that is much simpler to understand. This is the content of Section 3 where we construct free associative objects $\Omega(\tilde{A})$ and $\tilde{M}$ that satisfy the same covariance properties (Proposition 1). The standard picture and our picture are identical this far.

In Sections 4 and 5, we go from the associative picture to the commutative picture in two stages by passing to quotients of these free objects by imposing suitable relations. As we try to do that, the common picture breaks into two cases (Section 4.1). The first case leads to the standard picture of usual homological algebra which we are not considering here. So we continue the analysis with the 
second case. The covariance properties, since they hold in the free associative case, continue to hold after passing to quotients (Propositions 2 and 3).

In Section 6, we obtain Theorem 1 as a special case of Proposition 3. This case becomes important when we try to relate our construction to the Yang Baxter equation. We explain this connection in Section 7.

\section{The CATEgorical SEtTing}

In this section, we give the categorical framework for the algebras of forms considered in this paper. Observe that the algebras $A=k\left[x_{1}, \ldots, x_{n}\right], \tilde{A}=k\left\{x_{1}, \ldots, x_{n}\right\}$ and its quotient $A_{q}$ in Section 1, come equipped with the set of generators $S=$ $\left\{x_{1}, \ldots, x_{n}\right\}$. Further, the differential forms that we construct from these algebras depend on $S$ in an essential way. Hence, it is natural to do the following.

Definition 2.1. A $g$-algebra is a pair $(A, S)$, where $A=\langle S\rangle$ is an algebra over $k$ generated by the set $S$. The letter $g$ indicates that we have specified a set of generators.

2.1. The standard definitions. We first recall two definitions from [1, 2].

Definition 2.2. A graded $s$-differential algebra is a graded algebra

$$
\Omega=\oplus_{i \geq 0} \Omega^{i},
$$

with a degree 1 map $d: \Omega \rightarrow \Omega$ such that the $s$-Leibniz rule holds:

$$
d(u v)=d(u) v+s^{|u|} u d(v) .
$$

Here $|u|$ is the degree of $u$ with respect to the grading on $\Omega$. We will call $d$ a skewed derivation or a $s$-derivation for short.

Definition 2.3. Let $A$ be an algebra. A graded $s$-differential enveloping algebra of $A$ is a graded $s$-differential algebra such that $\Omega^{0}=A$.

2.2. The modified definitions. We now give the analogues of the above definitions in the category of $g$-algebras.

Definition 2.4. A graded $s$-differential $g$-algebra is a pair $(\Omega, S)$, where $\Omega$ is a graded $s$-differential algebra, $\left(\Omega^{0}, S\right)$ is a $g$-algebra and $d^{2}(S)=0$.

Lemma 1. For a graded s-differential g-algebra $(\Omega, S)$, if $s$ is a primitive $N$ th root of unity $(N \geq 2)$ and $\Omega=\langle S, d S\rangle$, then $d^{N}=0$.

Proof. We prove this result by induction. By definition, $d^{2}(S)=0$. Hence $d^{N}(S)=$ 0 , and $d^{N}(d S)=0$. This is the induction basis. Repeated application of the $s$ Leibniz rule in equation (2.1) gives

$$
d^{N}(u v)=\sum_{i=0}^{N} s^{i|u|}\left(\begin{array}{c}
N \\
i
\end{array}\right)_{s} d^{N-i}(u) d^{i}(v),
$$

where $\left(\begin{array}{c}N \\ i\end{array}\right)_{s}$ is the $s$-binomial coefficient. Note that if $s$ is a primitive $N$ th root of unity then $\left(\begin{array}{c}N \\ i\end{array}\right)_{s}=0$ for $1 \leq i \leq N-1$. And by induction, $d^{N}(u)=d^{N}(v)=0$. Hence $d^{N}(u v)=0$. Since $\Omega=\langle S, d S\rangle$, the result follows. 
Remark. Note that to get the conclusion $d^{N}=0$, it was enough to assume that $d^{N}(S)=0$. The stronger assumption $d^{2}(S)=0$, though unnecessary for Lemma 1 , simplifies the analysis done in this paper. Else for $x_{i} \in S$, one needs to introduce higher differential symbols $d^{2} x_{i}, d^{3} x_{i}$, and so on; see the paper of Kerner and Abramov [11] and the references therein for such considerations.

Definition 2.5. Let $(A, S)$ be a $g$-algebra. A graded $s$-differential enveloping $g$ algebra of $A$ is a graded $s$-differential $g$-algebra $(\Omega, S)$, with $\Omega^{0}=A$.

For a fixed $g$-algebra $(A, S)$, one can define the category of graded $s$-differential enveloping $g$-algebras of $A$.

\section{The free associative picture}

In this section, we perform the first step in the proof of Theorem 1, namely, prove it for the free associative case, see Proposition 1.

3.1. The algebra of forms $\Omega(\tilde{A})$. Let $V$ be the vector space over $k$ with basis $S=\left\{x_{1}, \ldots, x_{n}\right\}$.

Definition 3.1. Let $\tilde{A}=k\left\{x_{1}, \ldots, x_{n}\right\}$ be the free associative algebra generated by $S=\left\{x_{1}, \ldots, x_{n}\right\}$. Define $\Omega(\tilde{A})$ to be the universal object in the category of graded $s$-differential enveloping $g$-algebras of $\tilde{A}$. An explicit description is as below.

Remark. If $s=-1$ then the relevance of $S$ disappears and one can work with algebras instead of $g$-algebras. And $\Omega(\tilde{A})$ is the free differential envelope of $\tilde{A}$ and $d$ is a graded derivation of $\Omega(\tilde{A})$ satisfying $d^{2}=0$.

As an algebra,

$$
\Omega(\tilde{A})=k\left\{x_{1}, \ldots, x_{n}, d x_{1}, \ldots, d x_{n}\right\}
$$

is freely generated by $x_{1}, \ldots, x_{n}$ and the symbols $d x_{1}, \ldots, d x_{n}$. It is graded by the number of differential symbols. For example,

$$
x_{1} d x_{1} x_{2} d x_{2} d x_{2} \in \Omega(\tilde{A})
$$

is of degree 3. The differential $d: \Omega(\tilde{A}) \rightarrow \Omega(\tilde{A})$ is defined as follows:

Let $z_{k} \in\left\{x_{1}, \ldots, x_{n}, d x_{1}, \ldots, d x_{n}\right\}$ denote a generator. For a monomial $z=$ $z_{1} z_{2} \cdots z_{i} \in \Omega(\tilde{A})$, define

$$
d(z)=d\left(z_{1} \cdots z_{i}\right)=\sum_{k=1}^{i} s^{\left|z_{1} \cdots z_{k-1}\right|} z_{1} \cdots z_{k-1} d z_{k} z_{k+1} \cdots z_{i},
$$

where $d\left(x_{k}\right)=d x_{k}, d\left(d x_{k}\right)=0$ and $\left|z_{1} \cdots z_{k-1}\right|$ is the degree of the monomial $z_{1} \ldots z_{k-1}$, that is, it is the number of differential symbols that occur in $z_{1} \ldots z_{k-1}$. Note that the above definition is forced on us by equation (2.1). As an example,

$$
d\left(x_{1} d x_{2} x_{2} d x_{1} x_{1}\right)=d x_{1} d x_{2} x_{2} d x_{1} x_{1}+s x_{1} d x_{2} d x_{2} d x_{1} x_{1}+s^{2} x_{1} d x_{2} x_{2} d x_{1} d x_{1} .
$$

We set $d \tilde{A}=k\left\{d x_{1}, \ldots, d x_{n}\right\}$, the subalgebra of $\Omega(\tilde{A})$ that is freely generated by $d x_{1}, \ldots, d x_{n}$. It is then clear that $\Omega(\tilde{A})=\tilde{A} * d \tilde{A}$, the free product of $\tilde{A}$ and $d \tilde{A}$. 


\subsection{The universal bialgebra $\tilde{M}$.}

Definition 3.2. Let $\tilde{M}$ be the universal bialgebra that coacts on $V$ such that the coaction extends to $\tilde{A}$, making it a $\tilde{M}$ comodule algebra.

More explicitly, $\tilde{M}$ is the free associative algebra on the generators $t_{i j}$ for $1 \leq$ $i, j \leq n$ and the coproduct $\Delta: \tilde{M} \rightarrow \tilde{M} \otimes \tilde{M}$ sends the generator $t_{i j} \mapsto \sum_{k=1}^{n} t_{i k} \otimes$ $t_{k j}$. The coaction $\delta: \tilde{A} \rightarrow \tilde{M} \otimes \tilde{A}$ on the generating space $V$ sends the generator $x_{i} \mapsto \sum_{k=1}^{n} t_{i k} \otimes x_{k}$.

3.3. Relating $\Omega(\tilde{A})$ and $\tilde{M}$. The bialgebra $\tilde{M}$ can also be viewed as an universal object coacting on the algebra of forms $\Omega(\tilde{A})$ as below.

If we define a coaction $\delta: d \tilde{A} \rightarrow \tilde{M} \otimes d \tilde{A}$ that sends the generator $d x_{i} \mapsto$ $\sum_{k=1}^{n} t_{i k} \otimes d x_{k}$ then $d \tilde{A}$ is also a $\tilde{M}$ comodule algebra. Furthermore, $\delta$ extends uniquely to a coaction on $\Omega(\tilde{A})=\tilde{A} * d \tilde{A}$ making it a $\tilde{M}$ comodule algebra. One can directly check that the differential $d: \Omega(\tilde{A}) \rightarrow \Omega(\tilde{A})$ is a morphism of $\tilde{M}$ comodules. In other words, the following diagram commutes.

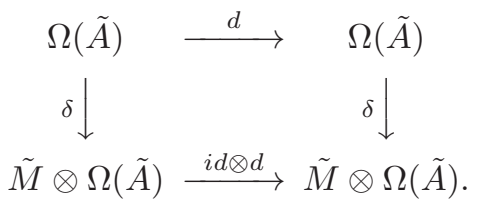

As already mentioned, $\delta$ in addition to being a comodule map is also a morphism of algebras.

The discussion in this section can be summarised as follows.

Proposition 1. Theorem 1 holds with $\Omega(\tilde{A})$ and $\tilde{M}$ instead of $\Omega\left(A_{q}\right)$ and $M_{q}$ respectively.

\section{IMPOSING RELATIONS}

In this section, we perform the second step in the proof of Theorem 1, namely, prove it for a quotient of the free associative case, see Proposition 2.

We are interested in the following deformation of the symmetric algebra $A$. Let

$$
A_{q}=k\left\{x_{1}, \ldots, x_{n}\right\} /\left(x_{i} x_{j}-q x_{j} x_{i} \text { for } i>j\right) .
$$

Setting $q=1$ gives us the symmetric algebra $A=k\left[x_{1}, \ldots, x_{n}\right]$. Note that $A_{q}$ is a quotient of the free associative algebra $\tilde{A}$. Our goal is to construct an algebra of forms $\Omega^{\prime}\left(A_{q}\right)$ and a bialgebra $M_{q}^{\prime}$ in this non-free situation. The idea is to obtain them as quotients of $\Omega(\tilde{A})$ and $\tilde{M}$, the free objects defined in Section 3, with the new differential $d$ and the new comodule map $\delta$ being the induced maps on the quotients. As before, we require that $\Omega^{\prime}\left(A_{q}\right)$ be a $M_{q}^{\prime}$ comodule algebra and the $s$-differential $d$ on $\Omega^{\prime}\left(A_{q}\right)$ be a morphism of $M_{q}^{\prime}$ comodules. In short, we want to pass to a quotient of the commutative diagram (3.1) of the previous section.

We will explain how these requirements point us to the relations that we must impose on $\Omega(\tilde{A})$ and $\tilde{M}$. Everything in this section is a formal consequence of our requirements. The only thing that was ad hoc was the particular deformation $A_{q}$ that we chose. 
4.1. Taking care of $d$. Applying $d$ to the relation $x_{i} x_{j}=q x_{j} x_{i}$ for $i>j$, we get

$$
d x_{i} x_{j}+x_{i} d x_{j}=q\left(d x_{j} x_{i}+x_{j} d x_{i}\right) \text { for } i>j .
$$

We will call this the connecting relation since it relates the function variables $x_{i}$ with the differentials $d x_{i}$. Next apply $d$ to the connecting relation $(C)$ to obtain

$$
(s+1) d x_{i} d x_{j}=q(s+1) d x_{j} d x_{i} \text { for } i>j .
$$

This yields two cases,

$$
s=-1 \quad \text { or } \quad d x_{i} d x_{j}=q d x_{j} d x_{i} \text { for } i>j .
$$

The first choice $s=-1$ corresponds to the standard picture of ordinary homological algebra $\left(d^{2}=0\right)$. This case when analysed further would lead to the usual quantum general linear group $G L_{q}(n)$. Since we would like $s$ to stay a free parameter, we choose the second alternative, that is, we impose $d x_{i} d x_{j}=q d x_{j} d x_{i}$ for $i>j$. For notational convenience, we set

$$
d A_{q}=k\left\{d x_{1}, \ldots, d x_{n}\right\} /\left(d x_{i} d x_{j}-q d x_{j} d x_{i} \text { for } i>j\right) .
$$

Note that $A_{q} \cong d A_{q}$ as algebras. Also observe that had we chosen $s=-1$ to start with we would have never seen this relation.

Definition 4.1. Let

$$
\Omega^{\prime}\left(A_{q}\right)=A_{q} * d A_{q} /(\text { connecting relation }(C)) .
$$

Note that by construction, $\Omega^{\prime}\left(A_{q}\right)$ is the universal object in the category of graded $s$-differential enveloping $g$-algebras of $A_{q}$.

4.2. Taking care of $\delta$. For this, we need to impose some relations on $\tilde{M}$. We begin by applying $\delta$ to the relation $x_{i} x_{j}=q x_{j} x_{i}$ for $i>j$. The left hand side looks as follows.

$$
\begin{aligned}
\delta\left(x_{i} x_{j}\right) & =\delta\left(x_{i}\right) \delta\left(x_{j}\right) \\
& =\sum_{k, l} t_{i k} t_{j l} \otimes x_{k} x_{l} \\
& =\sum_{k>l}\left(q t_{i k} t_{j l}+t_{i l} t_{j k}\right) \otimes x_{l} x_{k}+\sum_{k} t_{i k} t_{j k} \otimes x_{k}^{2} .
\end{aligned}
$$

The computation for the right hand side $\delta\left(q x_{j} x_{i}\right)$ is very similar. It can also be obtained from the above computation by interchanging $i$ and $j$ and adding a factor of $q$. Comparing the two sides, we get two relations on $\tilde{M}$.

(i) $\left(q t_{i k} t_{j l}+t_{i l} t_{j k}\right)=q\left(q t_{j k} t_{i l}+t_{j l} t_{i k}\right)$ for $i>j$ and $k>l$.

(ii) $t_{i k} t_{j k}=q t_{j k} t_{i k}$ for $i>j$. (column relations)

Definition 4.2. Define the bialgebra $M_{q}^{\prime}$ to be the quotient

$$
M_{q}^{\prime}=\tilde{M} /(\text { relations (i) and (ii)). }
$$

Strictly speaking, one still needs to check that the relations (i) and (ii) generate a coideal in $\tilde{M}$. This is a straightforward computation.

Note that by construction, $M_{q}^{\prime}$ is the universal bialgebra that coacts on $V$ such that the coaction extends to $A_{q}$, making it a $M_{q}^{\prime}$ comodule algebra.

Remark. The construction of $M_{q}^{\prime}$ from $A_{q}$ can be seen as a special case of a theorem of Manin, which says that for a quadratic algebra $A$, there is a universal bialgebra $A ! \bullet A$ that coacts on $A$, see parts (b) and (c) of the theorem in [13, Chapter 4]. In particular, we have

$$
M_{q}^{\prime}=A_{q}^{!} \bullet A_{q} .
$$


By appealing to Manin's theorem, one can avoid the above coideal computation.

4.3. Relating $\Omega^{\prime}\left(A_{q}\right)$ and $M_{q}^{\prime}$. Recall that in addition to the basic relation $x_{i} x_{j}=$ $q x_{j} x_{i}$ for $i>j$, we had imposed two more relations on $\Omega(\tilde{A})$, namely, the connecting relation $(C)$ and the relation $d x_{i} d x_{j}=q d x_{j} d x_{i}$ for $i>j$. These were obtained by successively applying $d$ to the basic relation. Hence, in view of the commutative diagram (3.1) at the end of Section 3, the map $\delta$ is automatically well-defined on these relations. In other words, applying $\delta$ to these relations would again lead to the same relations (i) and (ii) above. For $d x_{i} d x_{j}=q d x_{j} d x_{i}$, of course, we can also use that $A_{q} \cong d A_{q}$ as algebras and that $\delta$ respects this isomorphism.

The discussion in this section can be summarised as follows.

Proposition 2. Theorem 1 holds with $\Omega^{\prime}\left(A_{q}\right)$ and $M_{q}^{\prime}$ instead of $\Omega\left(A_{q}\right)$ and $M_{q}$ respectively.

\section{IMPOSING MORE RELATIONS}

In this section, we will perform the third step in the proof of Theorem 1, see Proposition 3.

From the standpoint of commutativity, the candidates $M_{q}^{\prime}$ and $\Omega^{\prime}\left(A_{q}\right)$ of Section 4 are not satisfactory. More precisely, the bialgebra $M_{q}^{\prime}$ when $q=1$ is not commutative; there are not enough relations. Similarly for $\Omega^{\prime}\left(A_{q}\right)$, the connecting relation $(C)$ does not allow us to move a $d x_{i}$ past a $x_{j}$. It is clear from this discussion that we need to pass to a further quotient.

At the end of Section 3, we had a complete picture. But it was not what we wanted. So in order to do the analysis in Section 4, we started with the deformed algebra $A_{q}$, an ad hoc choice. Now similarly at the end of Section 4, we again have a complete picture. But it is still not satisfactory. So we must make another ad hoc choice.

5.1. The twist relations. A natural thing to do is to add the following twist relations to $\Omega^{\prime}\left(A_{q}\right)$.

(1) $d x_{i} x_{j}=c_{i j}^{+} x_{j} d x_{i}+c_{i j}^{-} x_{i} d x_{j}$ for $i \neq j$.

(2) $d x_{k} x_{k}=u x_{k} d x_{k}$.

Here $c_{i j}^{+}, c_{i j}^{-}$and $u$ are constants to be determined. We will soon see that $u=s$. For a fixed $i>j$, the first relation really consists of two relations that refine the connecting relation $(C)$. The + superscript indicates that the constant $c_{i j}^{+}$is in some sense desirable. Similarly, the constant $c_{i j}^{-}$is undesirable. It is necessary to make things work but it must become zero when we specialise to $q=s=1$. The second relation is added to deal with the case $i=j$. Note that we have added the simplest possible relations to $\Omega^{\prime}\left(A_{q}\right)$ in order to make it more commutative. It is possible to consider more general and complicated relations. However it turns out that the relations we have added are sufficient to give something non-trivial and interesting and so we will stick to them.

5.2. Taking care of $d$. Now we play the same game as before. If we apply $d$ to the second twist relation then we see that $u=s$, that is,

$$
d x_{k} x_{k}=s x_{k} d x_{k} .
$$


The first twist relation is somewhat more interesting. Plugging it in the connecting relation $(C)$, we obtain

$$
c_{i j}^{+} x_{j} d x_{i}+\left(c_{i j}^{-}+1\right) x_{i} d x_{j}=q\left[c_{j i}^{+} x_{i} d x_{j}+\left(c_{j i}^{-}+1\right) x_{j} d x_{i}\right] .
$$

Also applying $d$ to the first twist relation gives

$$
s d x_{i} d x_{j}=c_{i j}^{+} d x_{j} d x_{i}+c_{i j}^{-} d x_{i} d x_{j} .
$$

With $i, j$ fixed and $i>j$, the above two equations give us 3 equations in the four unknowns $c_{i j}^{+}, c_{i j}^{-}, c_{j i}^{+}$and $c_{j i}^{-}$, namely,

$$
c_{i j}^{+}=q\left(c_{j i}^{-}+1\right), \quad q c_{j i}^{+}=c_{i j}^{-}+1, \quad \text { and } \quad s q=c_{i j}^{+}+q c_{i j}^{-} .
$$

Definition 5.1. Let

$$
\Omega^{\prime \prime}\left(A_{q}\right)=A_{q} * d A_{q} /(\text { relations }(1) \text { and }(2)),
$$

where $u=s$ and $c_{i j}$ are constants that satisfy equation (5.1). It is a graded $s$ differential enveloping $g$-algebra of $A_{q}$, but does not satisfy any universal property for $A_{q}$.

5.3. Taking care of $\delta$. Having dealt with $d$, we now take care of $\delta$. A routine computation similar to the one in Section 4.2 gives us the following relations on $\tilde{M}$.

(i) $c_{k l}^{+} t_{i k} t_{j l}+\left(c_{l k}^{-}-c_{i j}^{-}\right) t_{i l} t_{j k}=c_{i j}^{+} t_{j l} t_{i k}$ for $i \neq j, k \neq l$.

(ii) $\left(s-c_{i j}^{-}\right) t_{i k} t_{j k}=c_{i j}^{+} t_{j k} t_{i k}$ for $i \neq j . \quad$ (column relations)

(iii) $c_{k l}^{+} t_{i k} t_{i l}=\left(s-c_{l k}^{-}\right) t_{i l} t_{i k}$ for $k \neq l . \quad$ (row relations)

The first two relations are obtained by applying $\delta$ to the relation (1) while the third one is obtained by applying $\delta$ to the relation (2) in Section 5.1. Since the relation (1) is a refinement of the connecting relation $(C)$, the relations (i) and (ii) above respectively imply the relations (i) and (ii) of Section 4.2.

Definition 5.2. Define the bialgebra $M_{q}^{\prime \prime}$ as the quotient

$$
M_{q}^{\prime \prime}=\tilde{M} /(\text { relations (i),(ii) and (iii)). }
$$

As in the previous section, one can routinely check that the relations (i),(ii) and (iii) generate a coideal in $\tilde{M}$. This computation can be avoided by appealing to the FRT construction [7]. We explain this in Section 7.1.

5.4. Relating $\Omega^{\prime \prime}\left(A_{q}\right)$ and $M_{q}^{\prime \prime}$. Observe that $M_{q}^{\prime \prime}$ does not satisfy any universal property for $A_{q}$. However, from the discussion in this section, it does have an universal property for $\Omega^{\prime \prime}\left(A_{q}\right)$, as below.

Proposition 3. Theorem 1 holds with $\Omega^{\prime \prime}\left(A_{q}\right)$ and $M_{q}^{\prime \prime}$ instead of $\Omega\left(A_{q}\right)$ and $M_{q}$ respectively.

\section{Completing the proof of Theorem 1}

In this section, we explain how Theorem 1 is a special case of Proposition 3, see Lemma 3. 
6.1. The homogeneity condition. Recall that our goal was to construct a deformation $M_{q}$ of the commutative bialgebra $M$ in Definition 1.2. For that, we want to ensure that we have not added too many relations to $\tilde{M}$ in Definition 5.2. For example, the relation (i) is made up of four relations depending on how the indices $i$ and $j$, and $k$ and $l$ compare. For ease of comparison, we write them as follows always assuming that $i>j$ and $k>l$.

$$
\begin{array}{rlrlr}
t_{i k} t_{j l} & t_{i l} t_{j k} & & t_{j k} t_{i l} & t_{j l} t_{i k} \\
c_{k l}^{+}+\left(c_{l k}^{-}-c_{i j}^{-}\right) & = & & c_{i j}^{+} . \\
c_{j i}^{+} & = & c_{k l}^{+}+\left(c_{l k}^{-}-c_{j i}^{-}\right) . \\
\left.-c_{i j}^{-}\right)+ & = & c_{i j}^{+} . & \\
c_{j i}^{+} & & = & \left(c_{k l}^{-}-c_{j i}^{-}\right) & +
\end{array}
$$

In the above short hand notation, equation $\left(i^{\prime}\right)$ says that

$$
c_{k l}^{+} t_{i k} t_{j l}+\left(c_{l k}^{-}-c_{i j}^{-}\right) t_{i l} t_{j k}=c_{i j}^{+} t_{j l} t_{i k},
$$

and so on. We would only like two relations and not four. To get some linear dependence, we assume that for $k>l$ and $i>j$,

$$
c_{k l}^{+}=c_{i j}^{+}, \quad c_{k l}^{-}=c_{i j}^{-}, \quad c_{l k}^{+}=c_{j i}^{+}, \quad \text { and } \quad c_{l k}^{-}=c_{j i}^{-} .
$$

This may be regarded as a homogeneity condition on the variables. With this assumption, one readily checks that the relations $\left(\mathrm{i}^{\prime \prime \prime}\right)$ and $\left(\mathrm{i}^{\prime \prime \prime \prime}\right)$ are dependent on the first two. More precisely, $\left(\mathrm{i}^{\prime \prime}\right)=\left(\mathrm{i}^{\prime \prime \prime}\right)$ and $c_{j i}^{+}\left(\mathrm{i}^{\prime}\right)=c_{i j}^{+}\left(\mathrm{i}^{\prime \prime \prime \prime}\right)$. Also observe that by equation (5.1),

$$
q\left[\left(\mathrm{i}^{\prime}\right)+q\left(\mathrm{i}^{\prime \prime}\right)\right]=c_{k l}^{+}(\mathrm{i}),
$$

where (i) is the relation in Section 4.2.

Similarly, the relation (ii) seems to be made up of two relations, depending on how the indices $i$ and $j$ compare. However, it is actually only one relation. This becomes clear if we rewrite it as

$$
q=\frac{t_{i k} t_{j k}}{t_{j k} t_{i k}}=\frac{c_{i j}^{+}}{s-c_{i j}^{-}}=\frac{s-c_{j i}^{-}}{c_{j i}^{+}} \quad \text { for } i>j . \quad \text { (column relations) }
$$

The above equalities can be checked using equation (5.1). This also shows that the relation (ii) is completely equivalent to the relation (ii) of Section 4.2. The relation (iii) which we called the row relation is completely new. It was obtained by applying $\delta$ to the relation $d x_{k} x_{k}=s x_{k} d x_{k}$. Following the analogy with the relation (ii), one can rewrite it as

$$
q_{1}=\frac{t_{i k} t_{i l}}{t_{i l} t_{i k}}=\frac{c_{l k}^{+}}{s-c_{k l}^{-}}=\frac{s-c_{l k}^{-}}{c_{k l}^{+}} \quad \text { for } k>l . \quad \text { (row relations) }
$$

To summarise:

Lemma 2. Assuming the homogeneity condition $(\mathrm{H})$, the relations (i), (ii) and (iii) of Section 5.3 reduce to the relations $\left(\mathrm{i}^{\prime}\right),\left(\mathrm{i}^{\prime \prime}\right)$, (ii) and (iii) above. 
6.2. A special case. As mentioned earlier, the two constants $c_{j i}^{-}$and $c_{i j}^{-}$for $i>j$ with - superscripts are undesirable. But if we put $c_{j i}^{-}=c_{i j}^{-}=0$ then solving equation (5.1) gives $s=1$, which is unpleasant since we lose control over the parameter $s$. A good tradeoff is to put one of them equal to zero; say $c_{j i}^{-}=0$ for $i>j$. Then solving equation (5.1), we obtain

$$
c_{i j}^{+}=q, \quad c_{i j}^{-}=s-1 \text { and } c_{j i}^{+}=s q^{-1} .
$$

This solution is related to the Yang Baxter equation, see Proposition 5.

Lemma 3. For the choice of the constants $c_{i j}^{+}=q, c_{i j}^{-}=s-1, c_{j i}^{+}=s q^{-1}$ and $c_{j i}^{-}=0$ for $i>j$, the algebra of forms $\Omega^{\prime \prime}\left(A_{q}\right)$ and the quantum semigroup $M_{q}^{\prime \prime}$ in Definitions 5.1 and 5.2 specialise to $\Omega\left(A_{q}\right)$ and $M_{q}$ in Definitions 1.3 and 1.4 respectively.

Proof. The claim is straightforward for the algebra of forms. For the quantum semigroup, one uses Lemma 2 and checks that substituting the above solution in the relations $\left(\mathrm{i}^{\prime}\right),\left(\mathrm{i}^{\prime \prime}\right),(\mathrm{ii})$ and (iii) of Section 6.1 , we obtain the corresponding relations in Section 1.5.

\section{Connection to the Yang Baxter equation}

In this section, we explain how the bialgebra $M_{q}^{\prime \prime}$ in Proposition 3 can also be seen as a special case of the FRT construction. We will also explain the connection to the Yang Baxter equation (YB for short).

7.1. The quantum semigroup $M_{q}^{\prime \prime}$ as a special case. The existence of $M_{q}^{\prime \prime}$ can also be deduced from the following theorem which is central to the FRT construction.

Theorem 2. Let $W$ be a finite dimensional vector space and $c$ an endomorphism of $W \otimes W$. Then there exists a universal bialgebra $A(c)$, and a comodule map $\delta: W \rightarrow A(c) \otimes W$ such that the map $c$ is a morphism of comodules.

Let $W$ be a $n$ dimensional space with basis $e_{1}, e_{2}, \ldots, e_{n}$.

Definition 7.1. Define a linear map $c: W \otimes W \rightarrow W \otimes W$ using the twist relations of Section 5.1 as follows.

$$
\begin{aligned}
& c\left(e_{i} \otimes e_{j}\right)=c_{i j}^{+} e_{j} \otimes e_{i}+c_{i j}^{-} e_{i} \otimes e_{j} \text { for } i \neq j, \text { and } \\
& c\left(e_{k} \otimes e_{k}\right)=s e_{k} \otimes e_{k} .
\end{aligned}
$$

Here the $c_{i j}$ 's are constants that satisfy equation (5.1), with $q$ and $s$ as parameters.

Proposition 4. With $A(c)$ as in Theorem 2 for c as in Definition \%.1, and $M_{q}^{\prime \prime}$ as in Definition 5.2, we have

$$
A(c) \cong M_{q}^{\prime \prime} .
$$

Proof. The bialgebra $A(c)$ is the quotient of $\tilde{M}$ by the relations imposed by the condition that $c$ is a morphism of comodules. Similarly, $M_{q}^{\prime \prime}$ is the quotient of $\tilde{M}$ by the relations imposed by the condition that the coaction respects the twist relations (Section 5.3). Hence in view of Definition 7.1, we conclude that $A(c) \cong M_{q}^{\prime \prime}$. 
Remark. The bialgebra $A(c)$ in Theorem 2 can be defined using generators and relations, see [9, Definition VIII.6.2]. One can then check that for the $c$ chosen in Definition 7.1, these relations coincide with the relations (i), (ii) and (iii) in Section 5.3. This is a more direct way to prove the above proposition.

The main step in the proof of Theorem 2 is to show that the relations in the definition of $A(c)$ form a coideal, see the proof of [9, Lemma VIII.6.3]. This was precisely the computation that we omitted for $M_{q}^{\prime \prime}$.

7.2. The Yang Baxter equation. Let 1 denote the identity map of $W$ to itself. We say that a linear automorphism $c$ of $W \otimes W$ is a solution of the YB equation if

$$
(c \otimes 1)(1 \otimes c)(c \otimes 1)=(1 \otimes c)(c \otimes 1)(1 \otimes c)
$$

holds in the automorphism group of $W \otimes W \otimes W$.

Proposition 5. If the linear map $c$ in Definition 7.1 is a solution of the $Y B$ equation and the constants $c_{i j}$ satisfy the homogeneity condition $(\mathrm{H})$ in Section 6.1 then for $i>j$, either $c_{i j}^{-}=0$ or $c_{j i}^{-}=0$.

For the rest of the section, we will always assume that $c_{j i}^{-}=0$ for $i>j$. This was precisely the special case considered in Section 6.2. Recall that equation (5.1) then forces

$$
c_{i j}^{+}=q, \quad c_{i j}^{-}=s-1 \text { and } c_{j i}^{+}=s q^{-1} .
$$

Proposition 6. The linear map $c$ in Definition 7.1 , with the $c_{i j}$ 's as above is a solution of the $Y B$ equation iff $(s=q$ or $q=-1)$.

Furthermore, if $s=q$ then $c$ satisfies the quadratic relation $(c-q)(c+1)=0$.

Both the above propositions can be proved by direct computations. They are very similar to those in Kassel's book [9, pg 171]. Hence to get a solution to the YB equation, we either need to put $s=q$ or $q=-1$. In any case, we get a solution to the YB equation with one free parameter.

7.3. Braid groups and the Hecke algebra. We now explain the significance of the quadratic relation

$$
(c-q)(c+1)=0 .
$$

For every $n$, we have the braid group $B_{n}$ on $n$ strands. The Hecke algebra of type $A_{n-1}$ is a certain quotient of $k B_{n}$, the group algebra of $B_{n}$ over the field $k$. A solution $c$ of the YB equation can be used to construct a representation $W^{\otimes n}$ of the braid group $B_{n}$ for every $n$, as in [9, X.6.2]. And this representation factors through the corresponding Hecke algebra of type $A_{n-1}$ exactly when $c$ satisfies the above quadratic relation. Thus for $s=q$, our choice of $c$ gives a one parameter family of representations of the Hecke algebra.

\section{ACKNOWLEDGEMENTS}

I would like to thank K. Brown for many discussions on this subject and M. Dubois-Violette, M. Kapranov, R. Kerner, Yu. Manin and F. Ngakeu for useful correspondence. I would also like to thank the referee for useful comments regarding the exposition and for pointing out the references of Mayer and Spanier. This research was supported by the project G.0278.01 "Construction and applications of non-commutative geometry: from algebra to physics" from FWO Vlaanderen. 


\section{REFERENCES}

[1] M. Dubois-Violette and R. Kerner, Universal q-differential calculus and q-analog of homological algebra, Acta Math. Univ. Comenian. (N.S.) 65 (1996), no. 2, 175-188. 3, 5

[2] Michel Dubois-Violette, Generalized differential spaces with $d^{N}=0$ and the q-differential calculus, Czechoslovak J. Phys. 46 (1996), no. 12, 1227-1233, Quantum groups and integrable systems, I (Prague, 1996). 3, 5

$[3] \longrightarrow, d^{N}=0$ : generalized homology, K-Theory 14 (1998), no. 4, 371-404. 3

[4] _ Generalized homologies for $d^{N}=0$ and graded q-differential algebras, Secondary calculus and cohomological physics (Moscow, 1997), Contemp. Math., vol. 219, Amer. Math. Soc., Providence, RI, 1998, pp. 69-79. 3

[5] _ Lectures on differentials, generalized differentials and on some examples related to theoretical physics, Quantum symmetries in theoretical physics and mathematics (Bariloche, 2000), Contemp. Math., vol. 294, Amer. Math. Soc., Providence, RI, 2002, pp. 59-94. 3

[6] Michel Dubois-Violette and Richard Kerner, Universal $Z_{N}$-graded differential calculus, J. Geom. Phys. 23 (1997), no. 3-4, 235-246. 3

[7] L. D. Faddeev, N. Yu. Reshetikhin, and L. A. Takhtajan, Quantization of Lie groups and Lie algebras, Algebraic analysis, Vol. I, Academic Press, Boston, MA, 1988, pp. 129-139. 2, 10

[8] M.M. Kapranov, On the q-analog of homological algebra, arXiv:q-alg/9611005. 1, 2, 3

[9] Christian Kassel, Quantum groups, Springer-Verlag, New York, 1995. 1, 13

[10] Christian Kassel and Marc Wambst, Algèbre homologique des $N$-complexes et homologie de Hochschild aux racines de l'unité, Publ. Res. Inst. Math. Sci. 34 (1998), no. 2, 91-114. 3

[11] Richard Kerner and Viktor Abramov, On certain realizations of the q-deformed exterior differential calculus, Rep. Math. Phys. 43 (1999), no. 1-2, 179-194, Coherent states, differential and quantum geometry (Białowieża, 1997). 3, 6

[12] Yu. I. Manin, Some remarks on Koszul algebras and quantum groups, Ann. Inst. Fourier (Grenoble) 37 (1987), no. 4, 191-205. 1

[13] Quantum groups and noncommutative geometry, Université de Montréal Centre de Recherches Mathématiques, Montreal, QC, 1988. 1, 8

[14] _ Notes on quantum groups and quantum de Rham complexes, Teoret. Mat. Fiz. 92 (1992), no. 3, 425-450. 2

[15] W. Mayer, A new homology theory. I, II, Ann. of Math. (2) 43 (1942), 370-380, 594-605. 3

[16] Edwin H. Spanier, The Mayer homology theory, Bull. Amer. Math. Soc. 55 (1949), 102-112. 3

[17] Julius Wess and Bruno Zumino, Covariant differential calculus on the quantum hyperplane, Nuclear Phys. B Proc. Suppl. 18B (1990), 302-312 (1991), Recent advances in field theory (Annecy-le-Vieux, 1990). 1 